\title{
Relationship between chronic periodontitis and metabolic syndrome "Evaluation of periodontitis patients with metabolic syndrome and periodontitis patients without metabolic syndrome"
}

\author{
Ashwini Nareshchandra Walke* \\ MDS, Oral Pathologist, Riyadh, Saudi Arabia
}

\begin{abstract}
Background: The intention behind study was to scrutinize the periodontal status of patients with metabolic syndrome (Met.S) and periodontitis patients without Met.S and relate to general healthy subjects, to define whether the periodontal status was consistent in patients of Met.S.

Methods: A total of 150 subjects were scrutinized during study. Group one consisted of 50 healthy controls, group two consisted of 50 chronic periodontitis patients and group three consisted of 50 subjects with confirmed Met.S. Gingival Index, Bleeding Index (Ainamo \& Bay), Probing Pocket Depth (PPD), Clinical Attachment Level (CAL), were noted. Medical examination and blood investigations included measurement of Body-Mass Index (BMI), serum lipid profile, fasting blood glucose and blood pressure. Metabolic syndrome was defined according to NCEP ATP III definition. The results obtained were analyzed statistically using SPSS output.

Results: The results of the present study showed that the periodontal condition of group three patients were poor compared to group one \&two patients. The periodontal conditioned worsened in patients of metabolic syndrome.

Conclusion: Based on the results of our study, it can be concluded that periodontitis and metabolic syndrome were confounding the systemic effects of each other. Dentists should counsel their patients regarding the health hazards of metabolic syndrome and periodontitis and motivate them to maintain good oral hygiene and follow healthy life-style.
\end{abstract}

\section{Introduction}

According to WHO Report 2008, In India, 53\% of the deaths were due to Non-Communicable Diseases (NCD). Cardiovascular disease (CVDs) alone account for 24 percent of all deaths. These NCD include hypertension, heart diseases, stroke, diabetes, obesity, high cholesterol and diseases associated with tobacco use (smoking and chewing) like chronic bronchitis, Chronic obstructive pulmonary disease (COPD), cancer and excessive use of alcohol.

WHO have identified that most NCDs are the result of four particular lifestyle related behavioral risk factors like tobacco use, physical inactivity, unhealthy diet, and harmful use of alcohol that lead to four key metabolic/physiological changes e.g., raised blood pressure (BP), overweight/obesity, raised blood glucose and raised cholesterol levels.

The clinical cluster of hypertension, cardiovascular disease, hyperlipemia, hyperuricemia and type 2 Diabetes had been recognized by physicians for many decades, described as syndrome $\mathrm{X}$ and finally metabolic syndrome (Met.S) which was the subjects of this study [1-2].

The periodontal disease is a chronic, degenerative disease which is localized in the gingiva, periodontal ligament, cementum and alveolar bone. There is a known association between periodontitis and Met.S which warrants further investigations.

\section{Methods}

The present study was carried out in the Department of Oral Pathology and Microbiology of Sharad Pawar Dental College. The patients were selected from the Outpatient Department (OPD) of the department of Periodontics, Sharad Pawar Dental College and department of General Medicine, Achaya Vinoba Bhave Rural Hospital Sawangi, Meghe Wardha. Before the start of the study, an informed consent was obtained from all the study subjects. The study was approved by the Institutional Ethics Committee, Datta Meghe Institute of Medical Sciences, Wardha. Individuals of both genders of over 30 years of age (range 31 to 65 years) were used in the study. The total number of patients was 150 . Among them 100 were male patients and 50 were female patients. The study was performed on subjects, which were divided into three groups according to their periodontal status and presence or absence of Met.S.

1. Group I: Periodontally healthy subjects without systemic disorder. $(n=50)$.

${ }^{\star}$ Correspondence to: Ashwini Nareshchandra Walke, MDS, Oral Pathologist, Riyadh, Saudi Arabia, Email: ashwiniwalke2210@gmail.com

Key words: Chronic periodontitis, Metabolic syndrome (MS), Systemic diseases Received: July 12, 2018; Accepted: July 20, 2018; Published: July 25, 2018 
Walke AN (2018) Relationship between chronic periodontitis and metabolic syndrome "Evaluation of periodontitis patients with metabolic syndrome and periodontitis patients without metabolic syndrome"

2. Group II: Chronic Periodontitis patients without Met.S $(\mathrm{n}=50)$.

3. Group III: Chronic Periodontitis patients with Met.S $(\mathrm{n}=50)$ confirmed from their medical records.

Patients with history of smoking, patients on antibiotic therapy within previous three months, patients with dental prostheses, subjects with aggressive periodontitis and presence of any poorly controlled systemic condition that could have affected the progression of periodontitis other than Met.S were excluded from the study. They underwent a comprehensive general medical and dental examination. All individuals were examined in a standardized way with a mouth mirror and Williams's periodontal probe. All dental examinations were performed by one specially trained dentist. Bleeding Index (Ainamo \& Bay), Gingival index (Silness and Loe), Probing Pocket Depth (PPD) and Clinical Attachment Level (CEJ) The measurment was recorded on mesiobuccal, buccal, distobuccal and lingual sites of each tooth. The largest value recorded was used for further analysis.

Anthropometric measurements including weight, height was measured with the subjects wearing light clothing and no shoes. Height was measured in meters, using a hard ruler installed vertically and secured with a stable base. Body weight was measured using standard portable weighing machine $(1 \mathrm{~kg}$ was deducted from the weights recorded as an allowance for clothing). Body mass index was calculated. Blood pressure was measured manually using sphygmomanometer. Detailed clinical history was recorded, and clinical assessment was carried out in all the three groups. Blood samples were collected from all patients and analyzed for the serum parameters using enzymatic methods in the medical laboratory of Achaya Vinoba Bhave Rural Hospital Sawangi, Meghe Wardha. According to the NCEP ATP III definition, metabolic syndrome is present if three or more of the following five criteria are met: Body Mass Index (BMI), Obesity $>30$, blood pressure over $130 / 85 \mathrm{mmHg}$, triglyceride (TG) level over $150 \mathrm{mg} /$ dl, High-Density Lipoprotein (HDL) cholesterol level less than $40 \mathrm{mg} /$ $\mathrm{dl}$ (men) or $50 \mathrm{mg} / \mathrm{dl}$ (women) and fasting blood sugar over $110 \mathrm{mg} /$ dl. The data collected were analyzed statistically using SPSS software.

\section{Results and observations}

As shown in Table 1, the results of the present study showed that the BMI, blood pressure, fasting blood sugar level and serum lipid profiles were statistically significant between the groups (P value $<0.001$ ). When the general dental variables like Gingival index score, probing pocket depth, clinical attachment level and total number of sites with bleeding on probing were compared between the groups, group 3 showed both clinically and statistically higher values than group 1 and 2 (Tables 1 and 2). In Table 3 , the dental variables were compared according to the metabolic components using Pearson Correlation test (Table 3).

Statistically significant $(\mathrm{p}=0.00)$ highly positive correlation was observed between FBS and PPD, CAL, GI score, \% of site with bleeding when compared overall irrespective of groups. It means as the FBS increases the PPD, CAL, GI score, \% of sites with bleeding also increases.

Statistically significant $(\mathrm{p}=0.00)$ positive correlation was observed between TG and PPD, CAL, GI score, \% of site with bleeding when compared overall irrespective of groups. It means as the TG increases the PPD, CAL, GI score, \% of sites with bleeding also increases. Statistically significant $(p=0.00)$ positive correlation was observed between HDL and PPD, CAL, GI score, \% of site with bleeding when compared overall irrespective of groups. It means as the HDL increases the PPD, CAL, GI score, \% of sites with bleeding also increases. Statistically significant $(\mathrm{p}=0.00)$ positive correlation was observed between BMI and PPD, CAL, GI score, \% of site with bleeding when compared overall irrespective of groups. It means as the BMI increases the PPD, CAL, GI score, \% of sites with bleeding also increases. Statistically, non-significant correlation was observed between $\mathrm{BP}$ and PPD, CAL, GI score, $\%$ of sites with bleeding.

\section{Discussion}

Our findings were in accordance with, many studies which showed that, as the number of metabolic syndrome component increases the periodontitis severity also increases. Therefore, it could be hypothesized that with increase in number of Met.S factors in Met.S, the periodontitis parameters also increase. Also, highly positive correlation was observed between RBS and PPD [3-12]. Thus, it can be hypothesized that raised blood glucose levels were associated with increased in probing pocket depth.

The reasons for this finding could be that prolonged hyperglycemia causes the formation of AGE. Several cells, such as endothelial,

Table 1. Risk factors of all subjects

\begin{tabular}{|c|c|c|c|c|c|c|c|}
\hline \multicolumn{4}{|c|}{ Descriptive statistics } & \multicolumn{4}{|l|}{ ANOVA } \\
\hline Variables & $\begin{array}{l}\text { Mean } \pm \text { SD } \\
\text { Group 1 } \\
\mathrm{N}=50\end{array}$ & $\begin{array}{l}\text { Mean } \pm \text { SD } \\
\text { Group } 2 \mathrm{~N}=50\end{array}$ & $\begin{array}{l}\text { Mean } \pm \text { SD } \\
\text { Group } 3 \mathrm{~N}=50\end{array}$ & $\begin{array}{l}\text { Mean difference } \\
\text { Group } 1 \& 2\end{array}$ & $\begin{array}{l}\text { Mean difference } \\
\text { Group } 1 \& 3\end{array}$ & $\begin{array}{l}\text { Mean difference } \\
\text { Group } 2 \& 3\end{array}$ & P value \\
\hline BMI $\left(\mathrm{kg} / \mathrm{m}^{2}\right)$ & $21.46 \pm 1.948$ & $21.10 \pm 1.994$ & $24.21 \pm 1.877$ & 0.36 & -2.750 & -3.110 & $<0.001$ \\
\hline Sys. BP & $113.32 \pm 6.242$ & $127.92 \pm 8.098$ & $175.32 \pm 8.977$ & -14.600 & $-62.000^{*}-$ & -47.400 & .630 \\
\hline Dia. BP & $92.48 \pm 98.938$ & $78.34 \pm 10.392$ & $86.96 \pm 3.481$ & 14.140 & 5.520 & -8.620 & $<0.001$ \\
\hline $\mathrm{TG}(\mathrm{mg} / \mathrm{dl})$ & $123.16 \pm 23.868$ & $115.30 \pm 26.329$ & $173.20 \pm 15.683$ & 7.860 & -50.040 & $-57.900^{*}$ & $<0.001$ \\
\hline HDL (mg/dl) & $47.58 \pm 10.035$ & $51.54 \pm 11.906$ & $75.06 \pm 13.892$ & -3.960 & $-27.480^{*}$ & $-23.520^{*}$ & .187 \\
\hline FBS (mg/dl) & $110.70 \pm 11.553$ & $107.36 \pm 10.692$ & $218.74 \pm 40.977$ & 3.340 & -108.040 & -111.380 & $<0.001$ \\
\hline
\end{tabular}

Table 2. General dental variables

\begin{tabular}{|l|l|l|l|l|l|l|}
\hline Descriptive statistics & \multicolumn{3}{l|}{ ANOVA } \\
\hline Variables & $\begin{array}{l}\text { Mean } \pm \text { SD } \\
\text { Group 1 } \\
\mathrm{N}=50\end{array}$ & $\begin{array}{l}\text { Mean } \pm \text { SD } \\
\text { Group2 N }=50\end{array}$ & $\begin{array}{l}\text { Mean } \pm \text { SD } \\
\text { Group3 N }=50\end{array}$ & $\begin{array}{l}\text { Mean difference } \\
\text { Group 1\&2 }\end{array}$ & $\begin{array}{l}\text { Mean difference } \\
\text { Group 1\&3 }\end{array}$ & $\begin{array}{l}\text { Mean difference } \\
\text { Group 2\&3 }\end{array}$ \\
\hline GI & $.55 \pm .094$ & $1.78 \pm .177$ & $1.91 \pm .121$ & $-2.381^{*}$ & $-3.008^{*}$ & $-.627^{*}$ \\
\hline PPD $(\mathrm{mm})$ & $2.05 \pm .415$ & $4.43 \pm .643$ & $5.06 \pm .420$ & -2.761 & $-3.313^{*}$ & $-.553^{*}$ \\
\hline CAL $(\mathrm{mm})$ & $1.98 \pm .413$ & $4.74 \pm .677$ & $5.29 \pm .468$ & $-1.230^{*}$ & $-1.355^{*}$ & $-.125^{*}$ \\
\hline BOP $(\%)$ & $45.80 \pm 7.486$ & $67.84 \pm 6.100$ & $75.41 \pm 3.755$ & -22.04 & -29.56 & -0.001 \\
\hline
\end{tabular}


Walke AN (2018) Relationship between chronic periodontitis and metabolic syndrome "Evaluation of periodontitis patients with metabolic syndrome and periodontitis patients without metabolic syndrome"

Table 3. Co-relation of met.s with periodontitis in all groups

\begin{tabular}{|c|c|c|c|c|c|}
\hline & & PPD & CAL & GI & BLEEDING \\
\hline \multirow[t]{3}{*}{ RBS } & Pearson Correlation & $.521^{* *}$ & $.504^{* * *}$ & $.481^{* *}$ & $.511^{* *}$ \\
\hline & Sig. (2-tailed) & .000 & .000 & .000 & .000 \\
\hline & $\mathrm{N}$ & 150 & 150 & 150 & 150 \\
\hline \multirow{3}{*}{ TG } & Pearson Correlation & $.356^{* *}$ & $.372^{* *}$ & $.352^{* *}$ & $.368^{* *}$ \\
\hline & Sig. (2-tailed) & .000 & .000 & .000 & .000 \\
\hline & $\mathrm{N}$ & 150 & 150 & 150 & 150 \\
\hline \multirow{3}{*}{ HDL } & Pearson Correlation & $.489^{* *}$ & $.497^{* *}$ & $.466^{* *}$ & $.480^{* * *}$ \\
\hline & Sig. (2-tailed) & .000 & .000 & .000 & .000 \\
\hline & $\mathrm{N}$ & 150 & 150 & 150 & 150 \\
\hline \multirow{3}{*}{ BMI } & Pearson Correlation & $.305^{* *}$ & $.298^{* *}$ & $.281^{* *}$ & $.292^{* *}$ \\
\hline & Sig. (2-tailed) & .000 & .000 & .000 & .000 \\
\hline & $\mathrm{N}$ & 150 & 150 & 150 & 150 \\
\hline BP Pearson Correlation & & 0.092 & 0.037 & 0.028 & 0.029 \\
\hline Sig.(2-tailed) & & 0.579 & 0.808 & 0.350 & 0.350 \\
\hline $\mathrm{N}$ & & 150 & 150 & 150 & 150 \\
\hline
\end{tabular}

muscular, and immune cells, possess specific AGE receptors, called RAGE. AGEs also promote monocyte migration and increase endothelial permeability, fibroblasts, and muscular cell activity. In addition, AGEs can bind collagen, which if modified alters basement membrane structure. This leads to an inhibition of oxygen diffusion and tessellar oxygenation, diminished waste removal, altered immune mediators, and migration that causes an adulterated inflammatory response, which in turn causes increased damage to periodontal tissues, contributing to increase in PPD [3].

Our study revealed a low but positive correlation between BMI and clinical attachment loss. Recently, Han et al. concluded that the visceral fat area was the most appropriate indicator of obesity in relation to periodontitis and that obesity could act as a substantial risk factor for periodontitis. Although, the meta-analysis points to a positive association of obesity and periodontitis, the magnitude of the correlation is still not defined. This warrants further prospective studies to clarify the association [3].

However, few studies reported a non-association between periodontal disease and blood lipid levels Machado et al. 2005. In this study they suggest that abnormal glucose tolerance is a predisposing factor for diabetes mellitus, more as compared to impaired lipid metabolism and oxidized LDL cholesterol, does seem to be a risk indicator for periodontitis.

The association of obesity with periodontitis was explained as; Adipocytes of fat tissue show the ability to secrete adipocytokines, leptin, which shows a protective role against obesity. Also secrete other cytokines such as adiponectin and resistin. Serum adiponectin decreases in the presence of diabetes, obesity, IR , and CVD. Resistin shows a great proinflammatory role. During the development of periodontitis, in the attempt to remove causal agents, hyper inflammation is triggered, attracting numerous neutrophilic granulocytes, which, through the production of digestive enzymes during phagocytosis and killing, favor the production of ROS and the consequent constant release of inflammatory mediators with direct bacterial action damage on periodontal tissue [3].

Our study results showed, a significant correlation was observed between BP and periodontitis. The results of the study were in accordance with study done by Franek et al. 2009. But most of the studies found association with hypertension and periodontitis. However, data showed that periodontitis caused elevated levels of anti-cardiolipin antibodies. These might contribute to an increased risk for atherosclerosis in an individual. But our study lack to find this associations [13-16].

\section{The following hypotheses are suggested to explain the relationship between periodontitis and Met.S}

1. Cause-effect relationship- It has been reported that the association between periodontitis and Met.S could be bi-directional. The inflammatory markers in various components of Met.S can upregulate the periodontal inflammatory process and the persistent periodontal inflammation may worsen the inflammatory components of Met.S [3-9].

As a chronic inflammatory disease process, periodontitis can also have an adverse effect on Met.S. Subjects with periodontitis display an increment in the levels of various inflammatory markers. Products of periodontal inflammation may upgrade the levels of systemic cytokines, which may further enhance lipolysis. This may result in the increase of circulating TG and exacerbate IR [3-9].

Periodontitis and Met.S shares a common risk factors like excessive caloric intake, sedentary life-style and poor oral hygiene also includes hyperglycemia, obesity, dyslipidemia and elevated blood pressure [3-9].

2. Oxidative Stress could be a potential common link to explain the relationship between each component of Met.S and periodontitis. Since the metabolic alterations observed in that condition can cause an exacerbated host inflammatory response, metabolic syndrome individuals could have higher chances of undergoing tissue destruction in the presence of periodontal infection [12].

\section{Conclusion}

Periodontitis releases proinflammatory cytokines and ROS at the site of inflammation, leading to oxidative stress situation [16]. This will contribute to aggravate existing Met.S. Both of which act synergistically is a matter of debate.

Therefore, Met.S subjects should be recommended to go for frequent screening and periodontal treatment. Also, chronic periodontitis patients should be considered by multidisciplinary approach by physician, bearing in mind that the periodontal tissues exposed not only to local bacterial onslaught, but also systemic conditions damaging them through the same mechanisms provoking damage in other tissues, to control the severity of Met.S and restrict the morbidity and mortality attributed to the components of Met.S. 
Walke AN (2018) Relationship between chronic periodontitis and metabolic syndrome "Evaluation of periodontitis patients with metabolic syndrome and periodontitis patients without metabolic syndrome"

\section{References}

1. Aye (2014) Metabolic syndrome, J Metabolic syndrome 3: 4.

2. Chakma JK, Gupta S (2014) Review Article. Lifestyle and Non-Communicable Diseases: A double edged sword for future India. Indian Journal of Community Health 26.

3. Gurav AN (2014) The association of periodontitis and metabolic syndrome, The association of periodontitis and metabolic syndrome. Dental Research Journal 11.

4. Shimazaki Y, Saito T, Yonemoto KY, Kiyohara M, Yamashita IY (2007) Relationship of Metabolic Syndrome to Periodontal Disease in Japanese Women: The Hisayama Study. $J$ Dent Res 86: 271-275.

5. Khader Y, Khassawneh B, Obeidat B, Hamma M, El-Salem K, et al. (2008) Periodontal Status of Patients with Metabolic Syndrome Compared to Those Without Metabolic Syndrome. Journal of Periodontology 79: 2048-2053.

6. D'Aiuto F, Sabbah W, Netuveli G, Donos N, Hingorani AD, et al. (2008) Association of the Metabolic Syndrome with Severe Periodontitis in a Large U.S. Population-Based Survey. J Clin Endocrinol Metab 93: 3989-3994.

7. Kushiyama M, Shimazaki Y, Yamashita Y (2009) Relationship Between Metabolic Syndrome and Periodontal Disease in Japanese Adults. Journal of Periodontology 80: 1610-1615.

8. Nesbitt MJ, Reynolds MA, Shiau H, Choe K, Simonsick EM (2010) Association of periodontitis and metabolic syndrome in the Baltimore Longitudinal Study of Aging. $J$ Aging Clinical and Experimental Research 22: 238-242.
9. Young-Eun K, Jung-Eun H, Dai-Il P, Bo-Hyoung J, Kwang-Hak B (2011) The relationship between periodontitis and metabolic syndrome among a Korean nationally representative sample of adults. J Clin Periodontol 38: 781-786.

10. Bensley L, VanEenwyk J, Ossiander EM (2011) Associations of Self-Reported Periodontal Disease with Metabolic Syndrome and Number of Self-Reported Chronic Conditions. J Preventing chronic diseases 8: 3 .

11. Alhabashneh R, Khader Y, Herra Z, Assad F, et al. (2015) The association between periodontal disease and metabolic syndrome among outpatients with diabetes in Jordan. Journal of Diabetes \& Metabolic Disorders 14: 67.

12. Balagangadharan M, Subramaniam D, Swaminathan M, Saranyan R, Kathiresan VL, et al. (2015) International Journal of Research in Medical Sciences 3: 1257-1261.

13. Machado AC, Quirino MR, Nascimento LF (2005) Relation between chronic periodontal disease and plasmatic levels of triglyceridees, total cholesterol and fractions. Brazillian oral research 19: 284-289.

14. Dumitrescu AL, Zetu L, Teslaru S (2008) Metabolic syndrome and periodontal diseases. J intern med 46: 207-212.

15. Chaffee BW, Weston SJ (2010) Association between chronic periodontal disease and obesity: A systematic review and meta-analysis. J Periodontol 81: 1708-24. [Crossref]

16. Granados S, Nuri-El-Azem, Quiles JL, Perez-Lopez P, Gonzalez A, et al. (2012) Relationsip between cardiovascular risk factor and periodontal disease. Current Knowledge 193-216.

Copyright: $\odot 2018$ Walke AN. This is an open-access article distributed under the terms of the Creative Commons Attribution License, which permits unrestricted use, distribution, and reproduction in any medium, provided the original author and source are credited. 